\title{
Moser's lemma and the Darboux theorem
}

\author{
A. Lesfari \\ Department of Mathematics, Faculty of Sciences, University of Chouaïb Doukkali, B.P. 20, El-Jadida, Morocco \\ *Corresponding Author : Lesfariahmed@yahoo.fr
}

Copyright (C)2014 Horizon Research Publishing All rights reserved.

\begin{abstract}
In this paper we will give a proof of the classical Moser's lemma. Using it, we give a proof of the main Darboux theorem, which states that every point in a symplectic manifold has a neighborhood with Darboux coordinates.
\end{abstract}

Keywords Symplectic manifolds, Moser lemma, Darboux theorem

Mathematics Subject Classification (2010) : 53D05, 753D35

We will study in this note a theorem which plays a central role in symplectic geometry namely the Darboux theorem : the symplectic manifolds $(M, \omega)$ of dimension $2 m$ are locally isomorphic to $\left(\mathbb{R}^{2 m}, \omega\right)$. More precisely, if $(M, \omega)$ is a symplectic manifold of dimension $2 m$, then in the neighborhood of each point of $M$, there exist local coordinates $\left(x_{1}, \ldots, x_{2 m}\right)$ such that :

$$
\omega=\sum_{k=1}^{m} d x_{k} \wedge d x_{m+k} .
$$

In particular, there is no local invariant in symplectic geometry, analogous to the curvature in Riemannian geometry. The classical proof given by Darboux that bears his name is by induction on the dimension of the variety (see on this subject the excellent book by Arnold [1]). We will give an overview in remark 3. Another proof, based on a result of Moser [4], was given by Weinstein [5]. We will give in the following a proof of the Moser's lemma and then use this lemma to give a proof as direct as possible to the Darboux's theorem.

Lemma 1 Let $\left\{\omega_{t}\right\}, 0 \leq t \leq 1$, be a family of symplectic forms, differentiable in $t$. Then, for all $p \in M$, there exists a neighborhood $\mathcal{U}$ of $p$ and a function $g_{t}: \mathcal{U} \longrightarrow \mathcal{U}$, such that $: g_{0}^{*}=$ identity et $g_{t}^{*} \omega_{t}=\omega_{0}$.

Proof : Looking for a family of vector fields $X_{t}$ on $\mathcal{U}$ such that these fields generate locally a one-parameter group of diffeomorphisms $g_{t}$ with

$$
\frac{d}{d t} g_{t}(p)=X_{t}\left(g_{t}(p)\right), \quad g_{0}(p)=p
$$

First note that the form $\omega_{t}$ is closed (i.e., $d \omega_{t}=0$ ) as $\frac{d}{d t} \omega_{t}$ since

$$
d \frac{d}{d t} \omega_{t}=\frac{d}{d t} d \omega_{t}=0
$$

Therefore, by deriving the relationship $g_{t}^{*} \omega_{t}=\omega_{0}$ and using the Cartan homotopy formula

$$
L_{X_{t}}=i_{X_{t}} d+d i_{X_{t}}
$$

taking into account that $\omega_{t}$ depends on time, we obtain the expression

$$
\frac{d}{d t} g_{t}^{*} \omega_{t}=g_{t}^{*}\left(\frac{d}{d t} \omega_{t}+L_{X_{t}} \omega_{t}\right)=g_{t}^{*}\left(\frac{d}{d t} \omega_{t}+d i_{X_{t}} \omega_{t}\right)
$$

By Poincaré's lemma (in the neighborhood of a point, any closed differential form is exact), the form $\frac{\partial}{\partial t} \omega_{t}$ is exact in the neighborhood of $p$. In other words, we can find a form $\lambda_{t}$ such that :

$$
\frac{d}{d t} \omega_{t}=d \lambda_{t}
$$


Hence

$$
\frac{d}{d t} g_{t}^{*} \omega_{t}=g_{t}^{*} d\left(\lambda_{t}+i_{X_{t}} \omega_{t}\right)
$$

We want to show that for all $p \in M$, there exists a neighborhood $\mathcal{U}$ of $p$ and a function $g_{t}: \mathcal{U} \longrightarrow \mathcal{U}$, such that : $g_{0}^{*}=$ identity and $g_{t}^{*} \omega_{t}=\omega_{0}$, therefore such that :

$$
\frac{d}{d t} g_{t}^{*} \omega_{t}=0
$$

And by (1), the problem amounts to finding $X_{t}$ such that :

$$
\lambda_{t}+i_{X_{t}} \omega_{t}=0
$$

Since the form $\omega_{t}$ is nondegenerate, then the above equation is solvable with respect to the vector field $X_{t}$ and defines the family $\left\{g_{t}\right\}$ pour $0 \leq t \leq 1$. In local coordinates $\left(x_{k}\right)$ of the variety $M$ of dimension $2 m$, with $\left(\frac{\partial}{\partial x_{k}}\right)$ a basis of $T M$ and $\left(d x_{k}\right)$ the dual basis of $\left(\frac{\partial}{\partial x_{k}}\right), k=1, \ldots, 2 m$, we have

$$
\begin{aligned}
\lambda_{t} & =\sum_{k=1}^{2 m} \lambda_{k}(t, x) d x_{k}, \\
X_{t} & =\sum_{k=1}^{2 m} X_{k}(t, x) \frac{\partial}{\partial x_{k}}, \\
\omega_{t} & =\sum_{k, l=1}^{2 m} \omega_{k, l}(t, x) d x_{k} \wedge d x_{l}, \\
i_{X_{t}} \omega_{t} & =2 \sum_{l=1}^{2 m}\left(\sum_{k=1}^{2 m} \omega_{k, l} X_{k}\right) d x_{l} .
\end{aligned}
$$

We must therefore solve the system of equations in $x_{k}(t, x)$ according :

$$
\lambda_{l}(t, x)+2 \sum_{k=1}^{2 m} \omega_{k, l}(t, x) X_{k}(t, x)=0 .
$$

The form $\omega_{t}$ is nondegenerate, then the matrix $\left(\omega_{k}(t, x)\right)$ is nonsingular and therefore the above system has a unique solution. This determines the vector field $X_{t}$ and thus functions $g_{t}^{*}$ such that $: g_{t}^{*} \omega_{t}=\omega_{0}$, which completes the proof.

We are now able to give an easy direct proof of the Darboux's theorem.

Theorem 2 Any symplectic form on a manifold $M$ of dimension $2 m$ is locally diffeomorphic to the standard form on $\mathbb{R}^{2 m}$. In other words, if $(M, \omega)$ is a symplectic manifold of dimension $2 m$, then in the neighborhood of each point of $M$, there exist local coordinates $\left(x_{1}, \ldots, x_{2 m}\right)$ such that :

$$
\omega=\sum_{k=1}^{m} d x_{k} \wedge d x_{m+k} .
$$

Poof : Let $\left\{\omega_{t}\right\}, 0 \leq t \leq 1$, be a family of 2-differential forms which depends differentiably of $t$ and let

$$
\begin{aligned}
& \omega_{t}=\omega_{0}+t\left(\omega-\omega_{0}\right), \\
& \omega_{0}=\sum_{k=1}^{m} d x_{k} \wedge d_{m+k},
\end{aligned}
$$

where $\left(x_{1}, \ldots, x_{2 m}\right)$ are local coordinates on $M$. Note that these 2 -forms are closed. At $p \in M$, we have

$$
\omega_{t}(p)=\omega_{0}(p)=\omega(p)
$$

By continuity, we can find a small neighborhood of $p$ where the form $\omega_{t}(p)$ is nondegenerate. So the 2-forms $\omega_{t}$ are nondegenerate in a neighborhood of $p$ and independent of $t$ at $p$. In other words, $\omega_{t}$ are symplectic forms and by Moser's lemma, for all $p \in M$, there exists a neighborhood $\mathcal{U}$ of $p$ and a function $g_{t}: \mathcal{U} \longrightarrow \mathcal{U}$ such that : $g_{t}^{*}=$ identité and $g_{t}^{*} \omega_{t}=\omega_{0}$. Differentiating this relation with respect to $t$, we obtain (as in the proof of Moser's lemma),

$$
\begin{aligned}
\frac{d}{d t} g_{t}^{*} \omega_{t} & =0 \\
g_{t}^{*}\left(\frac{d}{d t} \omega_{t}+L_{X_{t}} \omega_{t}\right) & =0, \\
g_{t}^{*}\left(\frac{d}{d t} \omega_{t}+d i_{X_{t}} \omega_{t}\right) & =0 .
\end{aligned}
$$


Therefore,

$$
d i_{X_{t}} \omega_{t}=-\frac{d}{d t} \omega_{t}
$$

and since the form $\frac{d}{d t} \omega_{t}$ is exact in the neighborhood of $p$ (Poincaré's lemma), then

$$
d i_{X_{t}} \omega_{t}=d \theta_{t}
$$

where $\theta_{t}$ is a 1-differential form. In addition, $\omega_{t}$ being nondegenerate, the equation

$$
i_{X_{t}} \omega_{t}=\theta_{t}
$$

is solvable and determines uniquely the vector field $X_{t}$ depending on $t$. Note that for $t=1, \omega_{1}=\omega$ and for $t=0, \omega_{0}=\omega_{0}$ and also we can find $g_{1}^{*}$ such that $: g_{1}^{*} \omega=\omega_{0}$. Vector fields $X_{t}$ generate one-parameter families of diffeomorphisms $\left\{g_{t}\right\}, 0 \leq t \leq 1$. In other words, you can make a change of coordinates as :

$$
\omega=\sum_{k=1}^{m} d x_{k} \wedge d_{m+k},
$$

and the proof is completed.

Remark 3 As we reported earlier this note, we'll provide an overview of the classical proof given by Darboux. We proceed by induction on $m$. Suppose the result true for $m-1 \geq 0$ and show that it is also for $m$. Fix $x$ and let $x_{m+1}$ be a differentiable function on $M$ whose differential $d x_{m}$ is a nonzero point $x$. Let $X$ be the unique differentiable vector field satisfying the relation $i_{X} \omega=d x_{m+1}$. As this vector field does not vanish at $x$, then we can find a function $x_{1}$ in a neighborhood $\mathcal{U}$ of $x$ such that $X\left(x_{1}\right)=1$. Consider a vector field $Y$ on $\mathcal{U}$ satisfying the relation $i_{Y} \omega=-d x_{1}$. Since $d \omega=0$, then $L_{X} \omega=L_{Y} \omega=0$, according to the Cartan homotopy formula. therefore

$$
i_{[X, Y]} \omega=L_{X} i_{Y} \omega=L_{X}\left(i_{Y} \omega\right)-i_{Y}\left(L_{X} \omega\right)=L_{X}\left(-d x_{1}\right)=-d\left(X\left(x_{1}\right)\right)=0
$$

from which $[X, Y]=0$, since any point in the form $\omega$ is of rank equal to $2 m$. By the Recovery Theorem ${ }^{1}$, it follows that there exist local coordinates $x_{1}, x_{m+1}, z_{1}, Z_{2}, \ldots, z_{2 m-2}$ on a neighborhood $\mathcal{U}_{1} \subset \mathcal{U}$ of $x$ such that:

$$
X=\frac{\partial}{\partial x_{1}}, \quad Y=\frac{\partial}{\partial x_{m+1}} .
$$

Now consider the differential form

$$
\lambda=\omega-d x_{1} \wedge d x_{m+1}
$$

We have $d \lambda=0$ and

$$
i_{X} \lambda=L_{X} \lambda=i_{Y} \lambda=L_{Y} \lambda=0 .
$$

So $\lambda$ is expressed as a 2-differential form based only on variables $z_{1}, z_{2}, \ldots, z_{2 m-2}$. In particular, we have $\lambda^{m+1}=0$. Furthermore, we have

$$
0 \neq \omega^{m}=m d x_{1} \wedge d x_{m+1} \wedge \lambda^{m-1} .
$$

The 2 -form $\lambda$ is closed and of maximal rank (rank half) $m-1$ on an open set of $\mathbb{R}^{2 m-2}$. It is therefore sufficient to apply the induction hypothesis to $\lambda$.

Remark 4 Suppose that the variety $M$ is compact and connected. we show if

$$
\int_{M} \omega_{t}=\int_{M} \omega_{0}
$$

where $\left\{\omega_{t}\right\}, 0 \leq t \leq 1$ is a family of volume forms, then one can find a family of diffeomorphisms $g_{t}: M \longrightarrow M$, such that : $g_{0}^{*}=$ identity and $g_{t}^{*} \omega_{t}=\omega_{0}$. Indeed, just use a reasoning similar to Moser's lemma, provided to replace the Poincaré's lemma which is local, by the De Rham's theorem which is global. This means that a volume form $\omega$ on $M$ is exact if and only if $\int_{M} \omega=0$.

1. Let $X_{1}, \ldots, X_{r}$ differentiable vector fields on a manifold $M$ and $x \in M$. Assume that for all $k, l=1, \ldots, r,\left[x_{k}, X_{l}\right]=0$ and $X_{1}(x), \ldots, X_{r}(x)$ are linearly independent. We show that there is an open $\mathcal{U}$ of $M$ containing $x$ and a local coordinate system on $\mathcal{U}$ such that:

$$
\left.X_{1}\right|_{\mathcal{U}}=\frac{\partial}{\partial x_{1}}, \ldots,\left.X_{r}\right|_{\mathcal{U}}=\frac{\partial}{\partial x_{r}}
$$




\section{REFERENCES}

[1] Arnold, V.I. : Mathematical methods in classical mechanics. Springer-Verlag, Berlin-Heidelberg- New York, 1978.

[2] Lesfari, A. : Integrable systems and complex geometry. Lobachevskii Journal of Mathematics, Vol.30, No.4, 292-326 (2009).

[3] Lesfari, A. : Algebraic integrability : the Adler-van Moerbeke approach. Regul. Chaotic Dyn., Vol. 16, Nos.3-4, pp.187-209 (2011).

[4] Moser, J. K. : On the volume elements on a manifold. Trans. Amer. Math. Soc., 120, 286-294 (1965).

[5] Weinstein, A. : Symplectic manifolds and their lagrangian submanifolds. Adv. in Math., 6, 329-346 (1971). 